\title{
Longidorus poessneckensis Altherr, 1974 and L. piceicola Lišková, Robbins \& Brown, 1997 (Nematoda: Longidoridae): new records from Poland and the first description of the $L$. poessneckensis male and a bivulval female
}

\author{
Franciszek Wojciech Kornobis • Vlada Peneva
}

Received: 3 March 2011/Accepted: 30 June 2011

(C) The Author(s) 2011. This article is published with open access at Springerlink.com

\begin{abstract}
Longidorus poessneckensis Altherr, 1974 and L. piceicola Lišková, Robbins \& Brown, 1997 (Nematoda: Longidoridae) represent new records from Poland. These two species are described and illustrated together with a male and bivulval female of L. poessneckensis. In its general morphology and morphometrics, the male of $L$. poessneckensis is similar to the females, but has a spicule $100 \mu \mathrm{m}$ long and one adanal pair, two double and a row of six single ventromedian supplements. Comments on the differential diagnosis of $L$. poessneckensis and two morphologically related species, L. uroshis Krnjaić, Lamberti, Krnjaić, Agostinelli \& Radicci, 2000 and L. macrosoma Hooper, 1961 are given.
\end{abstract}

\section{Introduction}

Twelve species of Longidorus Micoletzky, 1922 have previously been reported from Poland. The most

F. W. Kornobis $(\bowtie)$

Department of Animal Morphology, Faculty of Biology, Adam Mickiewicz University, Umultowska 89,

61-614 Poznan, Poland

e-mail: franciszek.kornobis@amu.edu.pl

V. Peneva

Institute of Biodiversity and Ecosystem Research, Bulgarian Academy of Sciences, 2 Gagarin Street, 1113 Sofia, Bulgaria common species associated both with cultivated and wild growing plants are $L$. elongatus (de Man, 1876) Thorne \& Swanger, 1936, L. attenuatus Hooper, 1961, L. euonymus Mali \& Hooper, 1973 and L. leptocephalus Hooper, 1961 (Brzeski, 1963; Szczygieł, 1974; Brzeski, 1985; Szczygieł \& Brzeski, 1985). Other, less frequently occurring species are: L. balticus Brzeski, Peneva \& Brown, 2000, known only from Poland, L. caespiticola Hooper, 1961 and L. goodeyi Hooper, 1961 (see Szczygieł \& Brzeski, 1985), L. cylindricaudatus Kozłowska \& Seinhorst, 1979 (see Brzeski \& Winiszewska-Ślipińska, 1996), L. intermedius Kosłowska \& Seinhorst 1979 and L. macrosoma Hooper, 1961 (see Brzeski, 1985). More recent records include L. distinctus Lamberti, Choleva \& Agostinelli, 1983 and L. picenus Roca, Lamberti \& Da Costa, 1961 (see Szczygieł \& Zepp, 2004). No data on the morphology and morphometrics of the Polish populations of several species (L. caespiticola, L. goodeyi, L. cylindricaudatus, L. distinctus, L. macrosoma and L. picenus) are available in the above-mentioned references. During a recent survey of the family Longidoridae Thorne, 1935 in Poland, two previously unrecorded species were found: L. poessneckensis Altherr, 1974 and L. piceicola Lišková, Robbins \& Brown, 1997. Among the females of the former, an as yet undescribed male and a bivulval female were found. Data on morphology and morphometrics of both species are presented below. 


\section{Materials and methods}

Soil samples containing specimens of Longidorus were collected using a soil auger of $4 \mathrm{~cm}$ diameter. Nematodes were extracted from soil by the sieving and decanting method, heat-killed and preserved in cold TAF, with exception of the specimens of $L$. poessneckensis from the arboretum in Rogów, which were fixed in DESS (Yoder et al., 2006). Specimens were transferred to anhydrous glycerine using the Seinhorst method (Seinhorst, 1959). Identification and measurements were made using a Zeiss Axioskop 2 plus microscope. Measurements were taken with an eye graticule, except for body length which was measured using a map measurer. Photographs were taken using an Olympus BX 51 equipped with a digital camera.

In the description of the morphometrics, the ratios $\mathrm{d}$ (anterior to guide ring/body width at lip region) and $\mathrm{d}^{\prime}$ (body width at guide ring/body width at lip region) proposed by Brown et al. (1994) are used. Additionally, in the description of the bivulval female, ratios a1 and 2 are used; these were calculated using body width measured at the anterior and posterior vulva, respectively. All measurements are in micrometres.

\section{Longidorus poessneckensis Altherr, 1974}

Locality data: Material was collected from three localities in Poland (geographical coordinates in parentheses): near Górzyca $\left(52.28264^{\circ} \mathrm{N} ; 14.36871^{\circ} \mathrm{E}\right)$, population associated with Quercus robur L. and grasses; near Ustrzyki Dolne $\left(49.25694^{\circ} \mathrm{N} ; 22.30157^{\circ} \mathrm{E}\right)$, population associated with Carpinus betulus L. and Rubus sp.; the arboretum in Rogów $\left(51.83360^{\circ} \mathrm{N} ; 19.92320^{\circ} \mathrm{E}\right)$, where single specimens were found in sites close to each other but associated with different plants: Acer rubrum L. and cover plants; Betula alleghaniensis Britton and cover plants; and $C$. betulus (no cover plants). The specimens from Rogów are considered to represent a single population.

Description (Figs. 1, 2; Tables 1, 2)

Female (Fig. 1A-D)

Body open $\mathrm{C}$ to spiral in shape, with posterior third to half of body more curved. Lip region 6 high, continuous with rest of body. Cuticle 6 thick at guide
Fig. 1 Morphology of Longidorus poessneckensis. A. female, anterior end; B. female, anterior end with focus on amphidial fovea; C. female, vulval region; D. female, tail; E. male, posterior end; F. male, spicule; G. male, anterior end; H. bivulval female, vulval region; I. J3 juvenile, abnormal position of guide ring; J-M. tails of J1-J4 juveniles, respectively. Scale-bars: A-D,G,I,J-M, $20 \mu \mathrm{m}$; E,F,H, $50 \mu \mathrm{m}$

ring, 5 at mid-body and 9-14 on tail posterior to anus. Fine transverse cuticular striations present along entire body, 1.5-2 striations per $1 \mu \mathrm{m}$. Amphids with pouch-like fovea, 11-13 wide in widest part and visibly longer than wide; posterior limit indistinct (according to terminology proposed by Decraemer \& Coomans, 2007, following Chen et al., 1997, it is elongate-funnel shaped, i.e. code E4). Basal bulb occupies $25(21-33) \%$ of total pharynx length. Three gland nuclei present: 1 dorsal nucleus at 35 (27-42)\% $(\mathrm{n}=13)$ of pharyngeal bulb length and 2 ventrosublateral nuclei at $57(49-67) \%(\mathrm{n}=31)$ and 58 $(49-68) \%(\mathrm{n}=31)$. Vagina occupies $60-70 \%$ of corresponding body width; pars distalis vaginae and pars proximalis vaginae 22.4 (14-26) and 23.7 (20-28) long, respectively. Reproductive system amphidelphic, with equally developed genital branches. No sperm observed in genital tract. Tail almost hemispherical (majority of specimens) to bluntly conoidal, always visibly shorter than anal body width. Two caudal pores present on each side of body.

\section{Male (Fig. 1E-G)}

Morphology of anterior end similar to that of female; caudal region more strongly coiled ventrally. Tail short, rounded, with somewhat flattened tip. Cuticle 10-11 and 8 thick on dorsal and ventral part of tail, respectively. One adanal pair, two double and row of six single ventromedian supplements. Supplements arranged as follows: distance from anus to 1st pair, 17; 1 st to 2 nd pair, 16 ; 2nd to 3rd pair, 18 ; 3rd pair to 1st single, 35 ; between single ventral supplements as follows: 1 st to 2 nd, 39 ; 2nd to 3 rd, 48 ; 3 rd to 4 th, 38 ; 4 th to 5 th, $34 ; 5$ th to 6 th, 41 . Supplements extend $c .2$ from body contour.

Bivulval female (Fig. 1H)

Anomalous female found associated with Betula alleghaniensis. Morphometrics of bivulval female: $\mathrm{L}=8,815 ; \mathrm{a} 1=114.5 ; \mathrm{a} 2=117.5 ; \mathrm{b}=16.7 ; \mathrm{c}=$ $220.4 ; c^{\prime}=0.63 ; d=2.1 ; d^{\prime}=1.8 ; \quad V 1=52.2 \%$; 

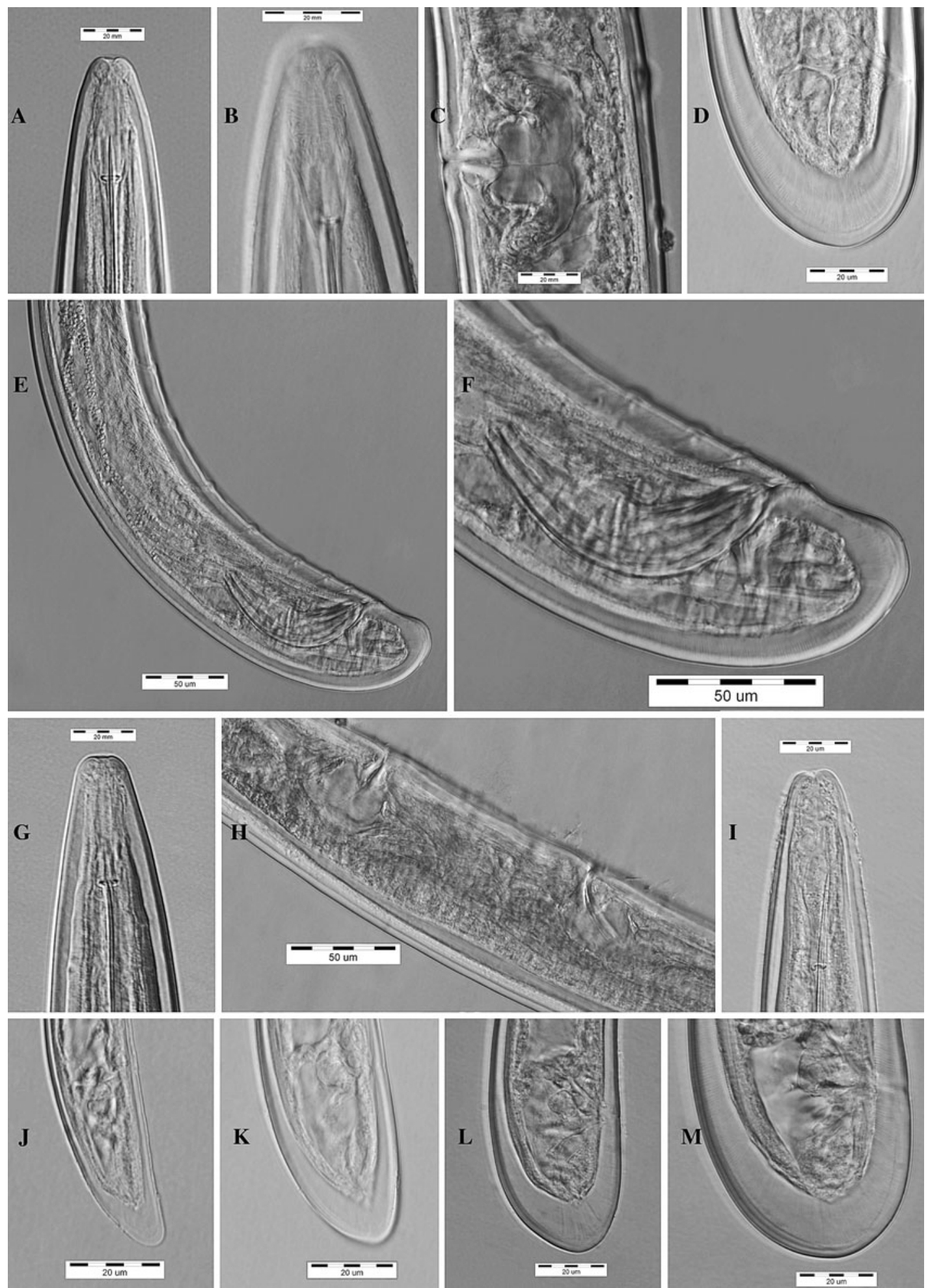


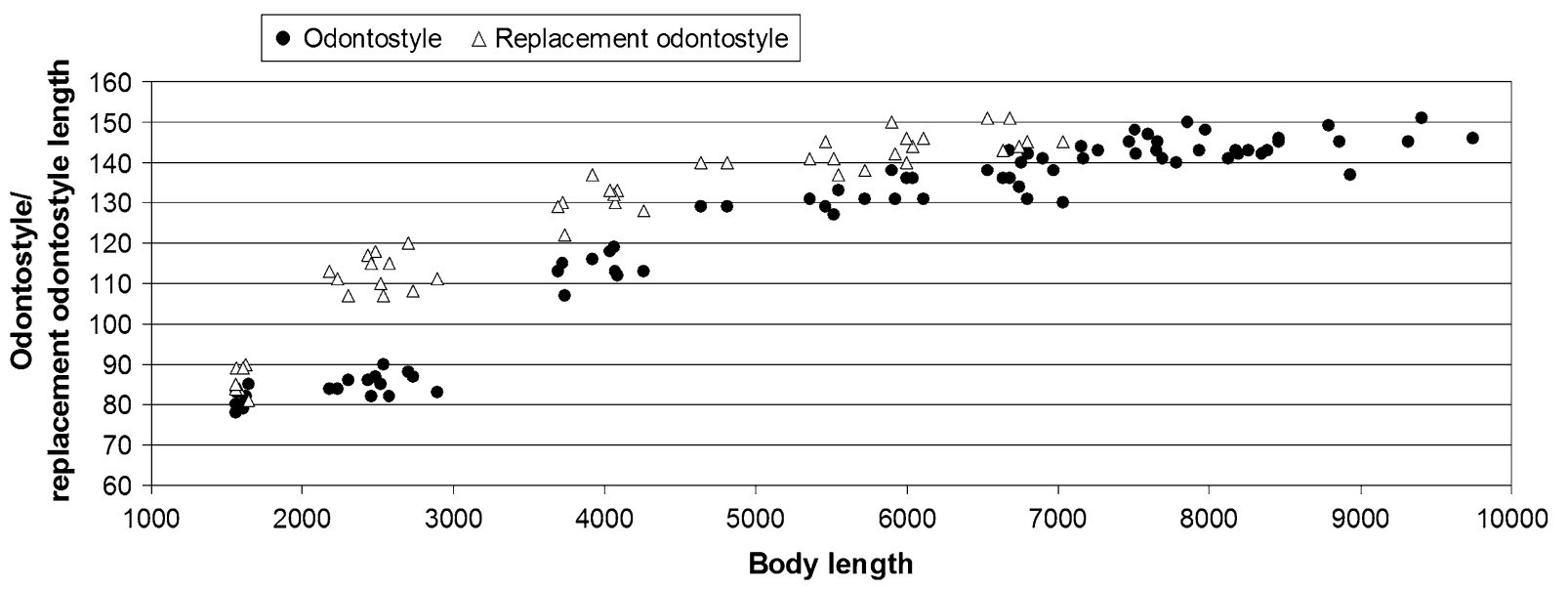

Fig. 2 Scatter plot of the functional and replacement odontostyle in relation to juvenile and females body length in a Longidorus poessneckensis population from Górzyca

Table 1 Morphometrics of adult Longidorus poessneckensis females from Poland

\begin{tabular}{|c|c|c|c|c|}
\hline \multirow{2}{*}{$\begin{array}{l}\text { Locality } \\
\text { Character }\end{array}$} & \multicolumn{2}{|l|}{ Górzyca } & \multirow{2}{*}{$\begin{array}{l}\text { Ustrzyki Dolne } \\
\text { Females } \\
\mathrm{n}=10\end{array}$} & \multirow{2}{*}{$\begin{array}{l}\text { Rogów } \\
\text { Females } \\
\mathrm{n}=4\end{array}$} \\
\hline & $\begin{array}{l}\text { Females } \\
\mathrm{n}=33\end{array}$ & $\begin{array}{l}\text { Male } \\
\mathrm{n}=1\end{array}$ & & \\
\hline Length & $\begin{array}{l}7,932 \pm 790.6 \\
(6,673-9,743)\end{array}$ & 9,379 & $\begin{array}{l}7,484 \pm 756.3 \\
(6,381-8,363)\end{array}$ & $\begin{array}{l}7,770 \\
(7,009-8,815)\end{array}$ \\
\hline $\mathrm{a}$ & $\begin{array}{l}104.3 \pm 7.7 \\
(93.9-119.5)\end{array}$ & 125.0 & $\begin{array}{l}97.8 \pm 7.6 \\
(84.2-106.1)\end{array}$ & $\begin{array}{l}97.0 \\
(86.9-114.5)\end{array}$ \\
\hline $\mathrm{b}$ & $\begin{array}{l}12.6 \pm 1.0 \\
(11.0-15.5)\end{array}$ & 13.4 & $\begin{array}{l}11.6 \pm 1.2 \\
(9.4-12.8)\end{array}$ & $\begin{array}{l}15.2 \\
(13.1-16.7)\end{array}$ \\
\hline $\mathrm{c}$ & $\begin{array}{l}215.8 \pm 16.9 \\
(179.1-256.4)\end{array}$ & 228.7 & $\begin{array}{l}180.7 \pm 22.9 \\
(153.9-237.2)\end{array}$ & $\begin{array}{l}185.8 \\
(164.2-220.4)\end{array}$ \\
\hline$c^{\prime}$ & $\begin{array}{l}0.64 \pm 0.04 \\
(0.56-0.70)\end{array}$ & 0.67 & $\begin{array}{l}0.73 \pm 0.06 \\
(0.61-0.80)\end{array}$ & $\begin{array}{l}0.70 \\
(0.63-0.76)\end{array}$ \\
\hline d & $\begin{array}{l}2.5 \pm 0.13 \\
(2.2-2.7)\end{array}$ & 2.6 & $\begin{array}{l}2.5 \pm 1.90 \\
(2.0-2.6)\end{array}$ & $\begin{array}{l}2.2 \\
(2.1-2.4)\end{array}$ \\
\hline $\mathrm{d}^{\prime}$ & $\begin{array}{l}2.1 \pm 0.12 \\
(1.8-2.3)\end{array}$ & 2.1 & $\begin{array}{l}1.9 \pm 0.1 \\
(1.67-2.06)\end{array}$ & $\begin{array}{l}1.92 \\
(1.8-2.1)\end{array}$ \\
\hline $\mathrm{V} /$ spicule & $\begin{array}{l}53.2 \pm 0.97 \\
(51.1-59.2)\end{array}$ & 100 & $\begin{array}{l}55.5 \pm 1.35 \\
(53.5-57.6)\end{array}$ & $\begin{array}{l}52.9 \\
(52.2-54.7)\end{array}$ \\
\hline Odontostyle & $\begin{array}{l}143.7 \pm 3.25 \\
(137-151)\end{array}$ & 143 & $\begin{array}{l}142.8 \pm 5.6 \\
(135-151)\end{array}$ & $\begin{array}{l}139.0 \\
(136-145)\end{array}$ \\
\hline Odontophore & $\begin{array}{l}92.2 \pm 6.1 \\
(81-105) \\
\mathrm{n}=32\end{array}$ & 102 & $\begin{array}{l}81.2 \pm 4.9 \\
(71-86)\end{array}$ & $\begin{array}{l}96 \\
\mathrm{n}=1\end{array}$ \\
\hline Total stylet & $\begin{array}{l}235.9 \pm 5.7 \\
(219-250) \\
\mathrm{n}=32\end{array}$ & 245 & $\begin{array}{l}224.0 \pm 5.8 \\
(216-237)\end{array}$ & $\begin{array}{l}232 \\
\mathrm{n}=1\end{array}$ \\
\hline
\end{tabular}


Table 1 continued

\begin{tabular}{|c|c|c|c|c|}
\hline \multirow{2}{*}{$\begin{array}{l}\text { Locality } \\
\text { Character }\end{array}$} & \multicolumn{2}{|l|}{ Górzyca } & \multirow{2}{*}{$\begin{array}{l}\text { Ustrzyki Dolne } \\
\text { Females } \\
\mathrm{n}=10\end{array}$} & \multirow{2}{*}{$\begin{array}{l}\text { Rogów } \\
\text { Females } \\
\mathrm{n}=4\end{array}$} \\
\hline & $\begin{array}{l}\text { Females } \\
\mathrm{n}=33\end{array}$ & $\begin{array}{l}\text { Male } \\
\mathrm{n}=1\end{array}$ & & \\
\hline Pharyngeal bulb length & $\begin{array}{l}157.6 \pm 11.4 \\
(135-178) \\
\mathrm{n}=30\end{array}$ & 177 & $\begin{array}{l}150.8 \pm 13.1 \\
(137-175) \\
\mathrm{n}=8\end{array}$ & $\begin{array}{l}139,155 \\
\mathrm{n}=2\end{array}$ \\
\hline Pharyngeal bulb width & $\begin{array}{l}28.9 \pm 1.85 \\
(26-33) \\
\mathrm{n}=30\end{array}$ & 33 & $\begin{array}{l}28.8 \pm 2.5 \\
(25-31) \\
\mathrm{n}=8\end{array}$ & $\begin{array}{l}29,29 \\
\mathrm{n}=2\end{array}$ \\
\hline Anterior end to guide ring & $\begin{array}{l}39.9 \pm 1.3 \\
(37-42)\end{array}$ & 42 & $\begin{array}{l}41.5 \pm 2.6 \\
(36-45)\end{array}$ & $\begin{array}{l}36.5 \\
(34-39)\end{array}$ \\
\hline Tail & $\begin{array}{l}36.8 \pm 2.8 \\
(31-43)\end{array}$ & 41 & $\begin{array}{l}41.7 \pm 4.4 \\
(34-47)\end{array}$ & $\begin{array}{l}42.0 \\
(40-45)\end{array}$ \\
\hline Hyaline tip & $\begin{array}{l}17.9 \pm 1.6 \\
(14-21)\end{array}$ & 17 & $\begin{array}{l}17.7 \pm 1.5 \\
(15-20)\end{array}$ & $\begin{array}{l}15.0 \\
(8-19)\end{array}$ \\
\hline \multicolumn{5}{|l|}{ Body width at } \\
\hline Lip region & $\begin{array}{l}15.8 \pm 0.8 \\
(14-18)\end{array}$ & 16 & $\begin{array}{l}16.6 \pm 0.8 \\
(16-18)\end{array}$ & $\begin{array}{l}16.3 \\
(16-17)\end{array}$ \\
\hline Guide ring & $\begin{array}{l}32.5 \pm 1.5 \\
(30-35)\end{array}$ & 33 & $\begin{array}{l}32.3 \pm 1.25 \\
(30-34)\end{array}$ & $\begin{array}{l}31.3 \\
(29-33)\end{array}$ \\
\hline Base of pharynx & $\begin{array}{l}63.3 \pm 3.6 \\
(58-71)\end{array}$ & 64 & $\begin{array}{l}61.8 \pm 4.3 \\
(55-70)\end{array}$ & $\begin{array}{l}60.5 \\
(57-63)\end{array}$ \\
\hline Vulva or mid-body & $\begin{array}{l}76.1 \pm 5.3 \\
(65-86)\end{array}$ & 75 & $\begin{array}{l}76.5 \pm 4.5 \\
(71-84)\end{array}$ & $\begin{array}{l}80.5 \\
(76-85)\end{array}$ \\
\hline Anus & $\begin{array}{l}57.8 \pm 2.9 \\
(54-67)\end{array}$ & 61 & $\begin{array}{l}57.2 \pm 2.5 \\
(55-62)\end{array}$ & $\begin{array}{l}60.3 \\
(58-63)\end{array}$ \\
\hline
\end{tabular}

Measurements $(\mu \mathrm{m})$ and ratios are in the form: mean \pm standard deviation (range). The value of ' $\mathrm{n}$ ' below the measurements indicates the number of specimens measured if different from that indicated in the heading. The standard deviation is not given when there are $<5$ measurements

$\mathrm{V} 2=53.5 \%$. Odontostyle, 138 ; anterior end to guide ring, 34; tail, 40; hyaline tip, 8; body width at: lip region, 16; guide ring, 9; base of pharynx, 62; vulva 1 , 77 ; vulva 2,75 ; anus, 63 ; distance from vulva 1 to vulva $2,118 \mu \mathrm{m}$. Detailed description of genital tract is not possible due to poor preservation of specimen; however, both vulvae appear unconnected. Additionally, two ovaries as well as two other structures resembling ovaries were observed. Remaining morphology similar to normal females from same population.

\section{Juveniles (Figs. 1J-M, 2; Table 2)}

General morphology similar to adult females, with differences mainly in body habitus and tail shape: $\mathrm{J} 1$ in form of a widely open $\mathrm{C}$, tail elongate, bluntly conoidal (Fig. 1J); J2 with shape of open C, tail elongate, almost cylindrical (Fig. $1 \mathrm{~K}$ ); J3 and $\mathrm{J} 4$ adopting a $\mathrm{J}$ - to $\mathrm{C}$-shape, tail of $\mathrm{J} 3$ similar to that of adults but more frequently bluntly conoidal (Fig. 1L), tail of $\mathrm{J} 4$ as in adults, hemispherical (Fig. 1M).

Single specimen (Fig. 1I) of J3 found with guide ring at 60 from anterior end; however, remaining morphometrics within ranges of normal J3. This specimen was not included in morphometrics of juveniles presented in Table 2.

\section{Remarks}

Longidorus poessneckensis, in addition to its typelocality in Germany (Altherr, 1974), has been reported from several other localities in Germany (Sturhan \& Loof, 2001), Slovakia (e.g. Lišková \& 
Table 2 Morphometrics of Longidorus poessneckensis juveniles from Górzyca, Poland

\begin{tabular}{|c|c|c|c|c|}
\hline Juvenile stage & $\begin{array}{l}\mathrm{J} 1 \\
\mathrm{n}=7\end{array}$ & $\begin{array}{l}\mathrm{J} 2 \\
\mathrm{n}=12\end{array}$ & $\begin{array}{l}\mathrm{J} 3 \\
\mathrm{n}=9\end{array}$ & $\begin{array}{l}\mathrm{J} 4 \\
\mathrm{n}=19\end{array}$ \\
\hline Length & $\begin{array}{l}1,592 \pm 34.1 \\
(1,553-1,643)\end{array}$ & $\begin{array}{l}2,503 \pm 207.6 \\
(2,178-2,891)\end{array}$ & $\begin{array}{l}3,954 \pm 197.9 \\
(3,695-4,257)\end{array}$ & $\begin{array}{l}5,968 \pm 662.4 \\
(4,640-7,034)\end{array}$ \\
\hline $\mathrm{a}$ & $\begin{array}{l}67.6 \pm 2.2 \\
(64.7-70.9)\end{array}$ & $\begin{array}{l}73.1 \pm 6.1 \\
(61.9-81.8)\end{array}$ & $\begin{array}{l}86.9 \pm 3.7 \\
(81.2 \pm 92.5)\end{array}$ & $\begin{array}{l}100.9 \pm 6.5 \\
(88.5 \pm 111.8)\end{array}$ \\
\hline $\mathrm{b}$ & $\begin{array}{l}4.5 \pm 0.1 \\
(4.4-4.7)\end{array}$ & $\begin{array}{l}5.9 \pm 0.4 \\
(5.4-6.7)\end{array}$ & $\begin{array}{l}7.4 \pm 0.3 \\
(6.9-7.8)\end{array}$ & $\begin{array}{l}9.8 \pm 0.9 \\
(7.6 \pm 11.1)\end{array}$ \\
\hline $\mathrm{c}$ & $\begin{array}{l}40.9 \pm 3.2 \\
(36.7-46.0)\end{array}$ & $\begin{array}{l}60.2 \pm 4.7 \\
(54.0-67.5)\end{array}$ & $\begin{array}{l}98.6 \pm 7.4 \\
(88.9-109.2)\end{array}$ & $\begin{array}{l}148.4 \pm 16.4 \\
(116.0 \pm 184.3)\end{array}$ \\
\hline $\mathrm{c}^{\prime}$ & $\begin{array}{l}2.11 \pm 0.21 \\
(1.84-2.39)\end{array}$ & $\begin{array}{l}1.42 \pm 0.11 \\
(1.28-1.63)\end{array}$ & $\begin{array}{l}0.95 \pm 0.07 \\
(0.83-1.03)\end{array}$ & $\begin{array}{l}0.77 \pm 0.06 \\
(0.64 \pm 0.89)\end{array}$ \\
\hline d & $\begin{array}{l}2.5 \pm 0.18 \\
(2.3-2.8)\end{array}$ & $\begin{array}{l}2.7 \pm 0.1 \\
(2.5-2.8)\end{array}$ & $\begin{array}{l}2.5 \pm 0.3 \\
(2.1-2.7)\end{array}$ & $\begin{array}{l}2.5 \pm 0.1 \\
(2.3 \pm 2.8)\end{array}$ \\
\hline $\mathrm{d}^{\prime}$ & $\begin{array}{l}1.8 \pm 0.2 \\
(1.7-2.0)\end{array}$ & $\begin{array}{l}2.1 \pm 0.11 \\
(2.0-2.3)\end{array}$ & $\begin{array}{l}1.9 \pm 0.2 \\
(1.7-2.3)\end{array}$ & $\begin{array}{l}2.0 \pm 0.1 \\
(1.87 \pm 2.20)\end{array}$ \\
\hline Odontostyle & $\begin{array}{l}81.0 \pm 2.45 \\
(79-85)\end{array}$ & $\begin{array}{l}85.3 \pm 2.5 \\
(80-92)\end{array}$ & $\begin{array}{l}114.0 \pm 3.6 \\
(107-119)\end{array}$ & $\begin{array}{l}132.7 \pm 3.4 \\
(127-138)\end{array}$ \\
\hline Replacement odontostyle & $\begin{array}{l}86.0 \pm 3.4 \\
(81-90)\end{array}$ & $\begin{array}{l}112.7 \pm 4.4 \\
(107-120)\end{array}$ & $\begin{array}{l}130.4 \pm 4.2 \\
(122-137)\end{array}$ & $\begin{array}{l}143.6 \pm 4.1 \\
(137-151)\end{array}$ \\
\hline Odontophore & $\begin{array}{l}57,57 \\
\mathrm{n}=2\end{array}$ & $\begin{array}{l}66.3 \pm 1.9 \\
(64-69) \\
n=6\end{array}$ & $\begin{array}{l}76.6 \pm 6.5 \\
(66-84) \\
\mathrm{n}=5\end{array}$ & $\begin{array}{l}84.0 \pm 5.0 \\
(76-91) \\
n=16\end{array}$ \\
\hline Total stylet & $\begin{array}{l}136,137 \\
\mathrm{n}=2\end{array}$ & $\begin{array}{l}152.5 \pm 3.3 \\
(148-158) \\
\mathrm{n}=6\end{array}$ & $\begin{array}{l}190.8 \pm 5.9 \\
(181-196) \\
\mathrm{n}=5\end{array}$ & $\begin{array}{l}216.7 \pm 6.4 \\
(205-225) \\
\mathrm{n}=16\end{array}$ \\
\hline Pharyngeal bulb length & $\begin{array}{l}85.6 \pm 2.2 \\
(82-88) \\
n=5\end{array}$ & $\begin{array}{l}104.2 \pm 5.9 \\
(95-114) \\
\mathrm{n}=11\end{array}$ & $\begin{array}{l}124.1 \pm 3.4 \\
(119-127) \\
\mathrm{n}=8\end{array}$ & $\begin{array}{l}145.6 \pm 8.9 \\
(129-160) \\
\mathrm{n}=17\end{array}$ \\
\hline Pharyngeal bulb width & $\begin{array}{l}14.8 \pm 0.8 \\
(14-16) \\
n=5\end{array}$ & $\begin{array}{l}18.6 \pm 1.3 \\
(17-21) \\
\mathrm{n}=11\end{array}$ & $\begin{array}{l}22 \pm 1.5 \\
(20-24) \\
\mathrm{n}=8\end{array}$ & $\begin{array}{l}26.7 \pm 1.7 \\
(22-30) \\
n=17\end{array}$ \\
\hline Anterior end to guide ring & $\begin{array}{l}21.1 \pm 0.4 \\
(21-22)\end{array}$ & $\begin{array}{l}25.2 \pm 0.4 \\
(25-26)\end{array}$ & $\begin{array}{l}29.3 \pm 1.2 \\
(27-31)\end{array}$ & $\begin{array}{l}35.2 \pm 1.4 \\
(32-38)\end{array}$ \\
\hline Genital primordium length & $\begin{array}{l}20.5 \pm 2.7 \\
(18-25) \\
\mathrm{n}=6\end{array}$ & $\begin{array}{l}27 \pm 1.5 \\
(25-30) \\
\mathrm{n}=10\end{array}$ & $\begin{array}{l}42 \pm 3.1 \\
(38-46) \\
n=5\end{array}$ & $\begin{array}{l}69.1 \pm 8.8 \\
(53-79) \\
n=9\end{array}$ \\
\hline Tail & $\begin{array}{l}39.1 \pm 2.8 \\
(35-43)\end{array}$ & $\begin{array}{l}41.7 \pm 3.2 \\
(37-47)\end{array}$ & $\begin{array}{l}40.2 \pm 2.6 \\
(38-46)\end{array}$ & $\begin{array}{l}40.3 \pm 3.1 \\
(35-46)\end{array}$ \\
\hline Hyaline tip & $\begin{array}{l}8.1 \pm 0.7 \\
(7-9)\end{array}$ & $\begin{array}{l}9.3 \pm 1.1 \\
(7-11)\end{array}$ & $\begin{array}{l}12.7 \pm 1.3 \\
(11-15)\end{array}$ & $\begin{array}{l}15.8 \pm 1.6 \\
(14-19)\end{array}$ \\
\hline \multicolumn{5}{|l|}{ Body width at } \\
\hline Lip region & $\begin{array}{l}8.4 \pm 0.5 \\
(8-9)\end{array}$ & $\begin{array}{l}9.5 \pm 0.5 \\
(9-10)\end{array}$ & $\begin{array}{l}11.9 \pm 0.9 \\
(11-13)\end{array}$ & $\begin{array}{l}14.1 \pm 0.7 \\
(13-15)\end{array}$ \\
\hline Guide ring & $\begin{array}{l}15.4 \pm 0.5 \\
(15-16)\end{array}$ & $\begin{array}{l}19.8 \pm 0.6 \\
(19-21)\end{array}$ & $\begin{array}{l}23.3 \pm 1.2 \\
(22-25)\end{array}$ & $\begin{array}{l}28.4 \pm 1.6 \\
(26-33)\end{array}$ \\
\hline
\end{tabular}


Table 2 continued

\begin{tabular}{lllll}
\hline Juvenile stage & $\mathrm{J} 1 \mathrm{n}=7$ & $\mathrm{~J} 2 \mathrm{n}=12$ & $\mathrm{~J} 3 \mathrm{n}=9$ & $\mathrm{~J} 4 \mathrm{n}=19$ \\
\hline Base of pharynx & $25.1 \pm 0.7$ & $35.2 \pm 2.6$ & $45.6 \pm 2.3$ & $56.1 \pm 4.9$ \\
& $(24-26)$ & $(31-40)$ & $(42-49)$ & $(46-65)$ \\
Mid-body & $23.6 \pm 1.0$ & $34.5 \pm 4.3$ & $45.6 \pm 2.5$ & $59.4 \pm 7.3$ \\
& $(22-25)$ & $(29-41)$ & $(42-50)$ & $(46-72)$ \\
Anus & $18.6 \pm 0.5$ & $29.4 \pm 2.6$ & $42.4 \pm 2.6$ & $52.3 \pm 3.8$ \\
& $(18-19)$ & $(25-34)$ & $(39-47)$ & $(45-57)$ \\
\hline
\end{tabular}

Measurements $(\mu \mathrm{m})$ and ratios are in the form: mean \pm standard deviation (range). The value of ' $n$ ' below the measurements indicates the number of specimens measured if different from that indicated in the heading. The standard deviation is not given when there are $<5$ measurements

Sturhan, 2000; Lišková \& Kumari, 2010), Austria (Tiefenbrunner \& Tiefenbrunner, 2004) and the Czech Republic (Kumari et al., 2009).

Morphometrically, populations from Poland are similar to each other, exhibiting only small difference in tail length (means $36.8 \mu \mathrm{m}$ in a population from Górzyca $v s 41.7$ and $42.2 \mu \mathrm{m}$ in populations from Ustrzyki Dolne and Rogów, respectively) and ratios based on tail length: c (mean 215.8 vs 180.7 and 185.8) and $\mathrm{c}^{\prime}$ (mean 0.64 vs 0.73 and 0.70 ).

The morphology of normal females is close to that given in the re-description given by Sturhan \& Loof (2001). In comparison with populations from other countries, those from Poland are most similar to populations from Germany (Sturhan \& Loof, 2001), the main difference being the odontostyle length (mean $133 \mu \mathrm{m}$ in German populations vs 143.7, 142.8 and $139.0 \mu \mathrm{m}$ in populations from Poland). Material from Poland is also similar to that from the Czech Republic (Kumari et al., 2009), but females are longer (means 7,932, 7,484 and 7,770 vs 6,851 $\mu \mathrm{m}$ ), more slender $(\mathrm{a}=104.3,97.8$ and 97.0 vs $\mathrm{a}=85.5)$ and have longer odontostyles (means 143.7, 142.8 and $139.0 \mu \mathrm{m} v s 128 \mu \mathrm{m}$ ). Moreover, the population from Górzyca has a higher c index (mean $215.8 \mathrm{vs}$ 177.3). Similarly, in comparison with populations from Austria (Tiefenbrunner \& Tiefenbrunner, 2004), and Slovakia (Lišková \& Sturhan, 2000; Lišková \& Kumari, 2010), specimens from Poland are longer (means 7,932, 7,484 and 7,770 $\mu \mathrm{m} v s$ 7,160, 6,500 and 6,690 $\mu \mathrm{m}$ ), more slender (means $\mathrm{a}=104.3,97.8$ and 97.0 vs $\mathrm{a}=818,90.8$ and 78), have longer odontostyles (means 143.7, 142.8 and $139.0 \mu \mathrm{m} v \mathrm{~s}$ $130,140.2$ and $127 \mu \mathrm{m})$ and higher c values (215.8, 180.7 and 185.8 vs 170.97, 154 and 158.6).
Sturhan \& Loof (2001) observed a slight increase in the tail length of subsequent juvenile stages. In Polish populations such a tendency has not been observed, tail length being about the same in all stages, similar to the results obtained by Kumari et al. (2009) from the Czech Republic.

The absence versus presence of males was used by Sturhan \& Loof (2001) as one of the morphological characters distinguishing $L$. poessneckensis from L. macrosoma Hooper, 1961. Because of the existence of the L. poessneckensis male, this 'trait' can no longer be used. However, the other traits proposed by those authors as differentiating these species, i.e. the shape of the lip region (rounded in L. poessneckensis $v s$ truncate in L. macrosoma), the structure of the cuticle on the tail (with thick, distinct median layers in L. macrosoma) and the shape of the J1 tail (conoidal vs subdigitate) are valid. Another species similar to L. poessneckensis, the differential diagnosis of which requires changes after the finding of the male, is L. uroshis Krnjaić, Lamberti, Krnjaić, Agostinelli \& Radicci, 2000. In the original description, Krnijać et al. (2000) did not compare these two species. Later, Lišková \& Sturhan (2002) distinguished them mainly on the basis of the presence and absence of males, respectively. This 'trait' can no longer be used, but these two species can be distinguished on the basis of spicule length-means 65.5 and $72 \mu \mathrm{m}$ (Krnijać et al., 2000; Lišková \& Sturhan, 2002) in L. uroshis vs $100 \mu \mathrm{m}$ in L. poessneckensis, the tail shape of the first-stage juvenile-subdigitate in $L$. uroshis vs bluntly conoidal in L. poessneckensis, and the amphidial fovea-bilobed at the base in L. uroshis $v s$ not bilobed in L. poessneckensis. For a discussion of other closely related species, as well as information on 
relationships based on molecular analyses, see Kumari et al. (2009).

The identification codes of the polytomous key published by Chen et al. (1997), based on the present data and other sources (Lišková \& Sturhan, 2000; Sturhan \& Loof, 2001; Tiefenbrunner \& Tiefenbrunner, 2004; Kumari et al., 2009; Lišková \& Kumari, 2010), are: A56 B1234 C34 D3 E4 F345 G12 H1 I12.

Anomalies in the female genital organs among Longidorus spp. are rather rare, although the phenomenon of bivulval female has been reported for other species of the genus, e.g. L. euonymus Mali \& Hooper, 1973 (see Barsi, 1994) and L. juvenilis Dalmasso, 1969 (see Širca et al., 2007).

According to the literature, L. poessneckensis occurs in moist to wet soils, particularly often associated with lowland riparian vegetation but also in wet woodlands in Germany, Slovakia, Austria and the Czech Republic (Sturhan \& Loof, 2001; Lišková \& Sturhan, 2000; Lišková, 2001; Tiefenbrunner \& Tiefenbrunner, 2004; for more references see Lišková \& Kumari, 2010). Our data are in general agreement with these observations; we have found populations in the same types of habitats, i.e. riparian and wet woodlands, but not in hilltop forests as reported by Lišková \& Kumari (2010).

\section{Longidorus piceicola Lišková, Robbins \& Brown, 1997}

Locality data: Specimens were collected from Rogów, Poland $\left(51.83360^{\circ} \mathrm{N} ; 19.92320^{\circ} \mathrm{E}\right), c .500 \mathrm{~m}$ from the site where L. poessneckensis occurred; the sample was taken from the soil around Carpinus betulus L. and no cover plants were present.
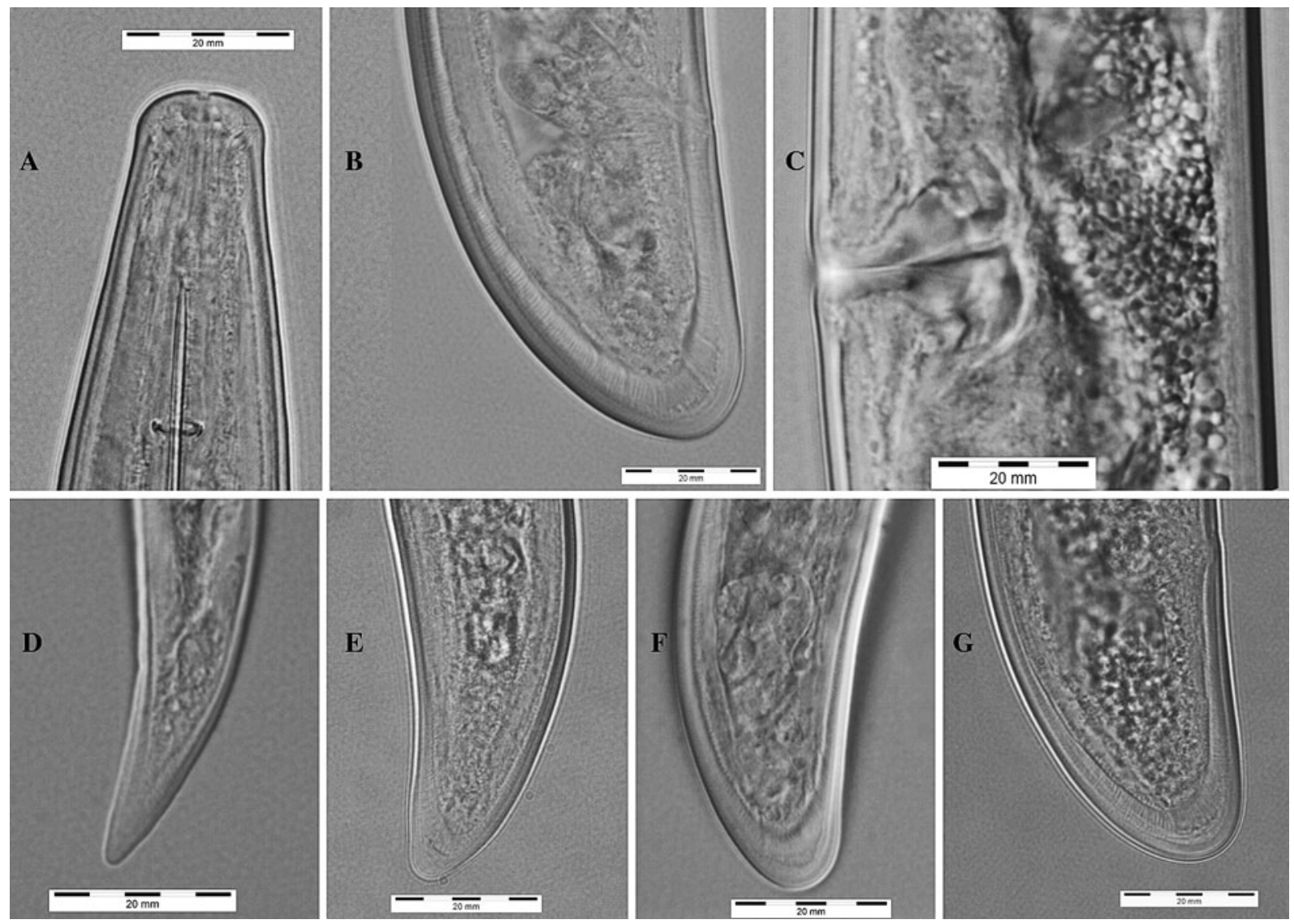

Fig. 3 Morphology of Longidorus piceicola. A. Female, anterior end; B. female, tail; C. female, vulval region; D-G. tails of J1-J4 juveniles, respectively. Scale-bars: $20 \mu \mathrm{m}$ 
Table 3 Morphometrics of females and juveniles of Longidorus piceicola from Poland

\begin{tabular}{|c|c|c|c|c|c|}
\hline Character & $\begin{array}{l}\text { Females } \\
\mathrm{n}=9\end{array}$ & $\begin{array}{l}\mathrm{J} 1 \\
\mathrm{n}=6\end{array}$ & $\begin{array}{l}\mathrm{J} 2 \\
\mathrm{n}=5\end{array}$ & $\begin{array}{l}\mathrm{J} 3 \\
\mathrm{n}=4\end{array}$ & $\begin{array}{l}\mathrm{J} 4 \\
\mathrm{n}=4\end{array}$ \\
\hline Length & $\begin{array}{l}6,477 \pm 468.6 \\
(5,457-7,093)\end{array}$ & $\begin{array}{l}1,446 \pm 68.1 \\
(1,368-1,529)\end{array}$ & $\begin{array}{l}2,117 \pm 160.4 \\
(1,913-2,334)\end{array}$ & $\begin{array}{l}2,883 \\
(2,714-3,073)\end{array}$ & $\begin{array}{l}4,249 \\
(3,956-4,503)\end{array}$ \\
\hline $\mathrm{a}$ & $\begin{array}{l}111.8 \pm 8.2 \\
(102.5-126.0)\end{array}$ & $\begin{array}{l}60.4 \pm 4.2 \\
(54.9-66.5)\end{array}$ & $\begin{array}{l}70.7 \pm 4.4 \\
(65.0-75.3)\end{array}$ & $\begin{array}{l}78.6 \\
(71.7-84.2)\end{array}$ & $\begin{array}{l}94.0 \\
(82.7-100.1)\end{array}$ \\
\hline $\mathrm{b}$ & $\begin{array}{l}11.8 \pm 0.7 \\
(10.4-12.8)\end{array}$ & $\begin{array}{l}4.6 \pm 0.1 \\
(4.5-4.8)\end{array}$ & $\begin{array}{l}5.7 \pm 0.4 \\
(5.3-6.3)\end{array}$ & $\begin{array}{l}6.6 \\
(5.6-7.3)\end{array}$ & $\begin{array}{l}8.1 \\
(7.6-9.3)\end{array}$ \\
\hline $\mathrm{c}$ & $\begin{array}{l}137.3 \pm 12.2 \\
(118.6-153.6)\end{array}$ & $\begin{array}{l}29.7 \pm 1.7 \\
(27.6-32.6)\end{array}$ & $\begin{array}{l}40.7 \pm 1.97 \\
(39.1-44.0)\end{array}$ & $\begin{array}{l}61.2 \\
(54.8-66.8)\end{array}$ & $\begin{array}{l}84.8 \\
(75.7-97.8)\end{array}$ \\
\hline $\mathrm{c}^{\prime}$ & $\begin{array}{l}1.09 \pm 0.09 \\
(0.96-1.24)\end{array}$ & $\begin{array}{l}3.03 \pm 0.27 \\
(2.56-3.36)\end{array}$ & $\begin{array}{l}2.25 \pm 0.15 \\
(2.04-2.45)\end{array}$ & $\begin{array}{l}1.63 \\
(1.54-1.70)\end{array}$ & $\begin{array}{l}1.36 \\
(1.19-1.51)\end{array}$ \\
\hline d & $\begin{array}{l}2.6 \pm 0.2 \\
(2.4-2.9)\end{array}$ & $\begin{array}{l}2.6 \pm 0.2 \\
(2.4-2.9)\end{array}$ & $\begin{array}{l}2.9 \pm 0.2 \\
(2.6-3.2)\end{array}$ & $\begin{array}{l}2.9 \\
(2.7-3.2)\end{array}$ & $\begin{array}{l}2.8 \\
(2.6-3.0)\end{array}$ \\
\hline $\mathrm{d}^{\prime}$ & $\begin{array}{l}1.6 \pm 0.1 \\
(1.56-1.67)\end{array}$ & $\begin{array}{l}1.6 \pm 0.1 \\
(1.56-1.67)\end{array}$ & $\begin{array}{l}1.8 \pm 0.01 \\
(1.77-1.80)\end{array}$ & $\begin{array}{l}1.8 \\
(1.7-2.0)\end{array}$ & $\begin{array}{l}1.7 \\
(1.7-1.8)\end{array}$ \\
\hline $\mathrm{V}$ & $\begin{array}{l}49.3 \pm 0.01 \\
(46.8-51.6)\end{array}$ & - & - & - & - \\
\hline Odontostyle & $\begin{array}{l}153.9 \pm 4.3 \\
(144-158)\end{array}$ & $\begin{array}{l}90.8 \pm 3.6 \\
(86-96)\end{array}$ & $\begin{array}{l}102.4 \pm 1.1 \\
(101-104)\end{array}$ & $\begin{array}{l}120.5 \\
(118-122)\end{array}$ & $\begin{array}{l}132.0 \\
(127-140)\end{array}$ \\
\hline Replacement odontostyle & - & $\begin{array}{l}98.8 \pm 4.2 \\
(92-105)\end{array}$ & $\begin{array}{l}116.4 \pm 1.5 \\
(114-118)\end{array}$ & $\begin{array}{l}131.3 \\
(127-140)\end{array}$ & $\begin{array}{l}151.5 \\
(148-160)\end{array}$ \\
\hline Odontophore & $\begin{array}{l}86.3 \pm 8.4 \\
(67-95) \\
\mathrm{n}=8\end{array}$ & $\begin{array}{l}48.2 \pm 3.6 \\
(45-54) \\
n=5\end{array}$ & $\begin{array}{l}55.6 \pm 3.2 \\
(52-59)\end{array}$ & $\begin{array}{l}67.0 \\
(64-72) \\
n=3\end{array}$ & $\begin{array}{l}78.3 \\
(70-85)\end{array}$ \\
\hline Total stylet & $\begin{array}{l}239.6 \pm 9.4 \\
(221-253)\end{array}$ & $\begin{array}{l}139.6 \pm 6.5 \\
(131-147) \\
\mathrm{n}=5\end{array}$ & $\begin{array}{l}158 \pm 3.9 \\
(154-163)\end{array}$ & $\begin{array}{l}187.7 \\
(182-194) \\
n=3\end{array}$ & $\begin{array}{l}211.0 \\
(201-225) \\
n=3\end{array}$ \\
\hline Pharyngeal bulb length & $\begin{array}{l}125.5 \pm 4.4 \\
(119-132) \\
\mathrm{n}=8\end{array}$ & $\begin{array}{l}65.7 \\
(60-71) \\
n=4\end{array}$ & $\begin{array}{l}83.8 \pm 2.95 \\
(80-88)\end{array}$ & $\begin{array}{l}99.8 \\
(91-109)\end{array}$ & $\begin{array}{l}109.7 \\
(102-115) \\
n=3\end{array}$ \\
\hline Pharyngeal bulb width & $\begin{array}{l}24.9 \pm 1.8 \\
(22-27) \\
n=8\end{array}$ & $\begin{array}{l}15.5 \\
(14-18) \\
\mathrm{n}=4\end{array}$ & $\begin{array}{l}17.6 \pm 1.1 \\
(16-19)\end{array}$ & $\begin{array}{l}19.8 \\
(18-22)\end{array}$ & $\begin{array}{l}21.3 \\
(19-24) \\
\mathrm{n}=3\end{array}$ \\
\hline Anterior end to guide ring & $\begin{array}{l}41.7 \pm 1.4 \\
(40-44)\end{array}$ & $\begin{array}{l}23.8 \pm 1.3 \\
(22-26)\end{array}$ & $\begin{array}{l}27.8 \pm 1.3 \\
(26-29)\end{array}$ & $\begin{array}{l}32.6 \\
(31-34)\end{array}$ & $\begin{array}{l}37.0 \\
(34-42)\end{array}$ \\
\hline Genital primordium length & - & $\begin{array}{l}27.4 \pm 2.7 \\
(23-30) \\
\mathrm{n}=5\end{array}$ & $\begin{array}{l}33.4 \\
(28-41) \\
n=4\end{array}$ & $\begin{array}{l}52.3 \\
(45-62) \\
n=3\end{array}$ & $\begin{array}{l}108 \\
(62-182)\end{array}$ \\
\hline Tail & $\begin{array}{l}47.3 \pm 3.4 \\
(43-52)\end{array}$ & $\begin{array}{l}48.8 \pm 4.5 \\
(42-54)\end{array}$ & $\begin{array}{l}52.0 \pm 2.0 \\
(49-54)\end{array}$ & $\begin{array}{l}47.3 \\
(44-51)\end{array}$ & $\begin{array}{l}50.5 \\
(44-56)\end{array}$ \\
\hline Hyaline tip & $\begin{array}{l}12.1 \pm 1.2 \\
(10-14)\end{array}$ & $\begin{array}{l}9.6 \pm 0.5 \\
(9-10)\end{array}$ & $\begin{array}{l}8.8 \pm 0.8 \\
(8-10)\end{array}$ & $\begin{array}{l}9.0 \\
8-10\end{array}$ & $\begin{array}{l}9.8 \\
(8-11)\end{array}$ \\
\hline \multicolumn{6}{|l|}{ Body width at } \\
\hline Lip region & $\begin{array}{l}15.4 \pm 0.5 \\
(15-16)\end{array}$ & $\begin{array}{l}9.0 \pm 0.0 \\
(9-9)\end{array}$ & $\begin{array}{l}9.6 \pm 0.6 \\
(9-10)\end{array}$ & $\begin{array}{l}11.3 \\
(10-12)\end{array}$ & $\begin{array}{l}13.5 \\
(12-15)\end{array}$ \\
\hline
\end{tabular}


Table 3 continued

\begin{tabular}{|c|c|c|c|c|c|}
\hline Character & $\begin{array}{l}\text { Females } \\
\mathrm{n}=9\end{array}$ & $\begin{array}{l}\mathrm{J} 1 \\
\mathrm{n}=6\end{array}$ & $\begin{array}{l}\mathrm{J} 2 \\
\mathrm{n}=5\end{array}$ & $\begin{array}{l}\mathrm{J} 3 \\
\mathrm{n}=4\end{array}$ & $\begin{array}{l}\mathrm{J} 4 \\
\mathrm{n}=4\end{array}$ \\
\hline Guide ring & $\begin{array}{l}27.0 \pm 1.2 \\
(26-29)\end{array}$ & $\begin{array}{l}14.7 \pm 0.5 \\
(14-15)\end{array}$ & $\begin{array}{l}17.2 \pm 1.1 \\
(16-18)\end{array}$ & $\begin{array}{l}20.3 \\
(20-21)\end{array}$ & $\begin{array}{l}23.5 \\
(22-25)\end{array}$ \\
\hline Base of pharynx & $\begin{array}{l}49.1 \pm 2.3 \\
(45-53)\end{array}$ & $\begin{array}{l}24.0 \pm 0.6 \\
(23-25)\end{array}$ & $\begin{array}{l}29.8 \pm 1.6 \\
(27-31)\end{array}$ & $\begin{array}{l}35.3 \\
(34-37)\end{array}$ & $\begin{array}{l}41.5 \\
(40-44)\end{array}$ \\
\hline Vulva or mid-body & $\begin{array}{l}58.1 \pm 4.5 \\
(51-64)\end{array}$ & $\begin{array}{l}24.0 \pm 1.6 \\
(22-26)\end{array}$ & $\begin{array}{l}30.0 \pm 2.4 \\
(26-32)\end{array}$ & $\begin{array}{l}36.8 \\
(35-39)\end{array}$ & $\begin{array}{l}45.5 \\
(40-52)\end{array}$ \\
\hline Anus & $\begin{array}{l}43.3 \pm 1.9 \\
(41-47)\end{array}$ & $\begin{array}{l}16.2 \pm 1.3 \\
(14-18)\end{array}$ & $\begin{array}{l}23.2 \pm 2.3 \\
(20-26)\end{array}$ & $\begin{array}{l}29.0 \\
(27-31)\end{array}$ & $\begin{array}{l}37.6 \\
(35-40)\end{array}$ \\
\hline
\end{tabular}

Measurements $(\mu \mathrm{m})$ and ratios are in the form: mean \pm standard deviation (range). The value of ' $\mathrm{n}$ ' below the measurements indicates the number of specimens measured if different from that indicated in the heading. The standard deviation is not given when there are $<5$ measurements

Description (Fig. 3; Table 3)

Females (Fig. 3A-C)

Habitus from J-shaped to spiral, more strongly coiled in posterior part of body. Cuticle c.3 thick at guide ring region and in mid-body, and 5-7 on tail posterior to anus. Fine transverse cuticle striations present along entire body, $c .2$ striations per $1 \mu \mathrm{m}$. Lip region 5 high, broadly rounded anteriorly, rounded laterally, almost continuous with rest of body. Amphids in most specimens with indistinct fovea, pocket-shaped when visible, symmetrically bilobed at base (according to terminology proposed by Decraemer \& Coomans, 2007). Pharyngeal bulb occupies 23 (20-24)\% of total pharynx length. Three gland nuclei present: dorsal nucleus located at $33(32-33) \%(n=3)$ of bulb length; 2 ventro-sublateral nuclei at 53 $(52-55) \%(\mathrm{n}=5)$ and $54(53-56) \%(\mathrm{n}=5)$. Vagina occupies $45-52 \%$ of corresponding body width; pars distalis vaginae and pars proximalis vaginae 14.3 (13-16) and 14.1 (13-15) long, respectively. Tail dorsally convex, flat or shallowly concave ventrally.

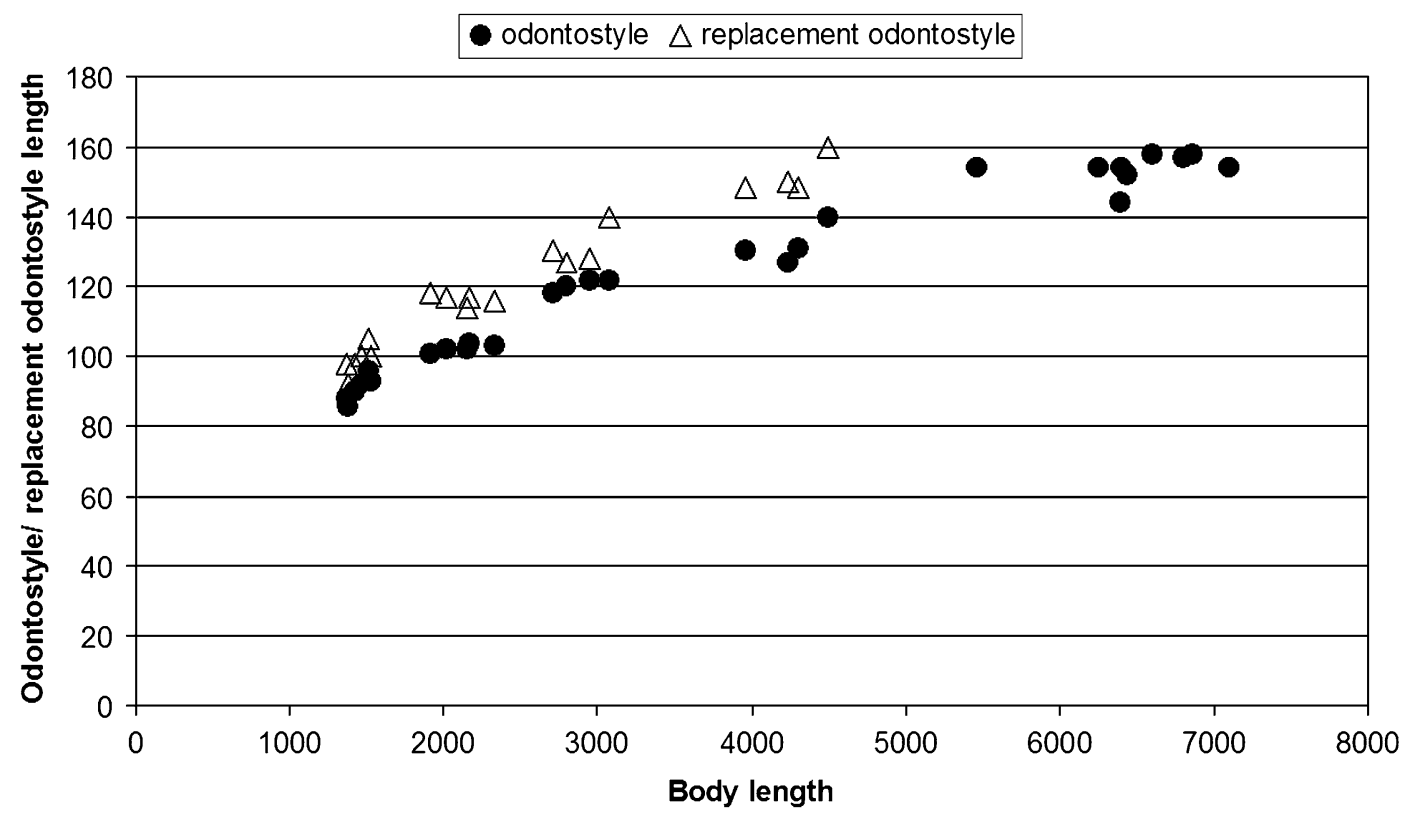

Fig. 4 Scatter plot of the functional and replacement odontostyle in relation to juvenile and female body length in a Longidorus piceicola population 
Male. Not found.

Juveniles (Figs. 3D-G, 4)

General morphology similar to adult females. Body habitus similar in all stages, open C- (arcuate) to J-shaped. Tail of all juvenile stages conical, but becoming more rounded in subsequent stages (Fig. 2D-G).

\section{Remarks}

Specimens of L. piceicola from Poland are similar to the type-population from Slovakia (Lišková et al., 1997), except for the longer (mean 6,477 vs $5,190 \mu \mathrm{m}$ ) and more slender (mean a value $111.8 \mathrm{vs}$ 94) body and longer tail (mean $47 v s 42 \mu \mathrm{m}$ ).

Barsi \& Lamberti (2001) described several L. piceicola populations from Bosnia and Herzegovina, Serbia and Montenegro. In comparison with those populations, the nematodes from Poland have a narrower lip region (mean $15.4 v s$ means within the range of 16-17 $\mu \mathrm{m}$ ) and a shorter odontostyle (mean $153.9 v s$ means within the range of $167-178 \mu \mathrm{m}$ ).

This species was reported in association with Picea abies L., Abies alba L. and Fagus sylvatica L. (Lišková et al., 1997; Barsi \& Lamberti, 2001), and the finding of this species in Poland in association with Carpinus betulus extends the geographical and plant association ranges.

Acknowledgement This work was supported by Dean of The Faculty of Biology, Adam Mickiewicz University Poznań, Poland. Project number PB WB 04/2009.

Open Access This article is distributed under the terms of the Creative Commons Attribution Noncommercial License which permits any noncommercial use, distribution, and reproduction in any medium, provided the original author(s) and source are credited.

\section{References}

Altherr, E. (1974). Nématodes de la nappe phréatique du réseau fluvial de Saale (Turinge) II. Limnologica, 9, 81-132.

Barsi, L. (1994). Bivulval females of Longidorus euonymus, Xiphinema diversicaudatum and X. vuittenezi (Nematoda: Dorylaimida). Nematologia Mediterranea, 22, 271-272.

Barsi, L., \& Lamberti, F. (2001). Morphometric variation, juvenile stages of Longidorus piceicola Liskova et al., 1997 (Nematoda: Longidoridae) from the former territory of Yugoslavia. Russian Journal of Nematology, 9, 77-83.
Brown, D. J. F., Grunder, J., Hooper, D. J., Klinger, J., \& Kunz, P. (1994). Longidorus arthensis sp. nov. (Nematoda: Longidoridae) a vector of cherry rosette disease caused by a new nepovirus in cherry trees in Switzerland. Nematologica, 40, 133-149.

Brzeski, M. W. (1963). [Nematodes of the City Park in Skierniewice. I. Soil nematodes.] Fragmenta Faunistica, 10, 441-461. (In Polish).

Brzeski, M. W. (1985). [Materials for the recognition of domestic nematodes (Nematoda) — plant parasites. 7. Ten species new for Poland.] Fragmenta Faunisitca, 29, 275-288. (In Polish).

Brzeski, M. W., Peneva, V., \& Brown, D. J. F. (2000). Longidorus balticus sp. nov. (Nematoda: Longidoridae) from coastal sand dunes in northeast Poland. Annales Zoologici, 50, 321-325.

Brzeski, M. W., \& Winiszewska-Ślipińska, G. (1996). Preliminary list of the soil inhabiting nematodes of the Białowieża primeval forest. Fragmenta Faunistica, 39, 245-257.

Chen, Q. W., Hooper, D. J., Loof, P. A. A., \& Xu, J. (1997). A revised polytomous key for the identification of species of the genus Longidorus Micoletzky, 1922 (Nematoda: Dorylaimoidea). Fundamental and Applied Nematology, 20, 15-28.

Decraemer, W., \& Coomans, A. (2007). Revision of some species of the genus Paralongidorus sensu Siddiqi et al. (1993), with a discussion on the relationships within the family Longidoridae (Nematoda: Dorylaimida). Nematology, 9, 643-662.

Krnjaić, D., Lamberti, F., Krnjaić, S., Agostinelli, A., \& Radicci, V. (2000). Three new longidorids (Nematoda: Dorylaimida) from Montenegro. Nematologia Mediterranea, 28, 235-248.

Kumari, S., Decraemer, W., Traversa, D., \& Lišková, M. (2009). Molecular and morphological delineation of Longidorus poessneckensis Altherr, 1974 (Nematoda: Dorylaimida). European Journal of Plant Pathology, 123, 125-137.

Lišková, M. (2001). Longidoridae (Nematoda: Dorylaimida) in natural grasslands and fluvial plains in the Slovak Republic. Helminthologia, 38, 47-50.

Lišková, M., \& Kumari, S. (2010). The notes on the occurrence of Longidorus poessneckensis Altherr, 1974 (Nematoda: Dorylaimida) in Slovak Republic. Helminthologia, 47, 264-268.

Lišková, M., Robbins, R. T., \& Brown, D. J. F. (1997). Descriptions of three new Longidorus species from Slovakia (Nemata: Longidoridae). Journal of Nematology, 29, 336-348.

Lišková, M., \& Sturhan, D. (2000). Occurrence and ecology of Longidoridae (Nematoda: Dorylaimida) in floodplain forest in the Slovak Republic. Helminthologia, 37, 113-117.

Lišková, M., \& Sturhan, D. (2002). Longidorus uroshis Krnjaić, et al., 2000 (Nematoda, Dorylaimida) in the Slovak Republic. Helminthologia, 39, 233-236.

Seinhorst, J. W. (1959). A rapid method for the transfer of nematodes from fixative to anhydrous glycerin. Nematologica, 4, 67-69. 
Sturhan, D., \& Loof, P. A. A. (2001). Redescription of Longidorus poessneckensis Altherr, 1974 (Nematoda: Dorylaimida). Russian Journal of Nematology, 9, 43-49.

Širca, S., Stare, B. G., Pleško, I. M., Marn, M. V., \& Urek, G. (2007). First record of Longidorus juvenilis and L. leptocephalus (Nematoda: Dorylaimida) in Slovenia and their morphometrical and ribosomal DNA sequence analysis. Russian Journal of Nematology, 15, 1-8.

Szczygieł, A. (1974). Plant parasitic nematodes associated with strawberry plantations in Poland. Zeszyty Problemowe Postepów Nauk Rolniczych, 154, 1-132.

Szczygieł, A., \& Brzeski, M. W. (1985). Distribution of Longidoridae, Xiphinemidae and Trichodoridae. In: Alphey, T. J. W. (Ed.) Atlas of plant parasitic nematodes of
Poland. Dundee: Scottish Crop Research Institute, pp. 1-32.

Szczygiel, A., \& Zepp, A. (2004). The association of plant parasitic nematodes with fruit crops in Poland as related to some soil properties. Fragmenta Faunistica, 47, 7-33.

Tiefenbrunner, A., \& Tiefenbrunner, W. (2004). Longidoridae (Nematoda: Dorylaimida) from the rhizosphere of the wild growing grape (Vitis vinifera $\mathrm{ssp}$. silvestris) in the riparian woods of the rivers Danube and March in Austria. Helminthologia, 41, 45-53.

Yoder, M., De Ley, I. T., King, I. W., Mundo-Ocampo, M., Mann, J., Blaxter, M., Poiras, L., \& De Ley, P. (2006). DESS: A versatile solution for preserving morphology and extractable DNA of nematodes. Nematology, 8, 367-376. 